\title{
Early Process Development: The Wyeth Approach
}

\author{
Michael K. O’Brien*, Michael Kolb*, Karen Sutherland, Kevin McCoy, and Anthony Pilcher
}

\begin{abstract}
The paper describes our approach to early process development in the context of the new Wyeth productivity model to deliver two New Molecular Entities every year. As a consequence, the number of compounds in Predevelopment and Phase 0 increased fourfold, the cycle time from Discovery to the IND was shortened by 6 months, and the quantities for the Phase- 0 batch increased to include Phase I supply. Our new way of working to meet this paradigm without a major increase in headcount is delineated in this article.
\end{abstract}

Keywords: Development $\cdot$ Process $\cdot$ Scale-up $\cdot$ Strategy

\section{Introduction}

At least one of us can remember a time when a compound from Discovery entering the development pipeline was a feast for process chemists. Our highly refined synthesis and optimization skills would be brought to bear on each and every compound that entered the development portfolio. The objective to develop a process that was fully optimized and ready to be commercialized tomorrow was blind to the fact that most compounds never made it to the market. How times have changed!!

Over the last five years, Wyeth has pursued a productivity model that has as its objective the submission of two NDAs for New Molecular Entities every year. To support this goal, 60 compounds advanced into Phase 0, and 48 Investigational New Drugs (IND) were filed during that time. This corresponds to a four-fold increase in annual output by Discovery over their performance five years prior, and, as a result, an equal increase in workload for the chemists and engineers in Chemical Development. In

*Correspondence: Dr. M. Kolb, Dr. M.K. O’Brien Wyeth Research

401 N. Middletown Road

Pearl River, NY 10965

USA

Tel.: +1 845602 3601(3493) or +1 8456026301

Fax: +18456025189

E-Mail: kolbm@wyeth.com or obrienm4@wyeth.com

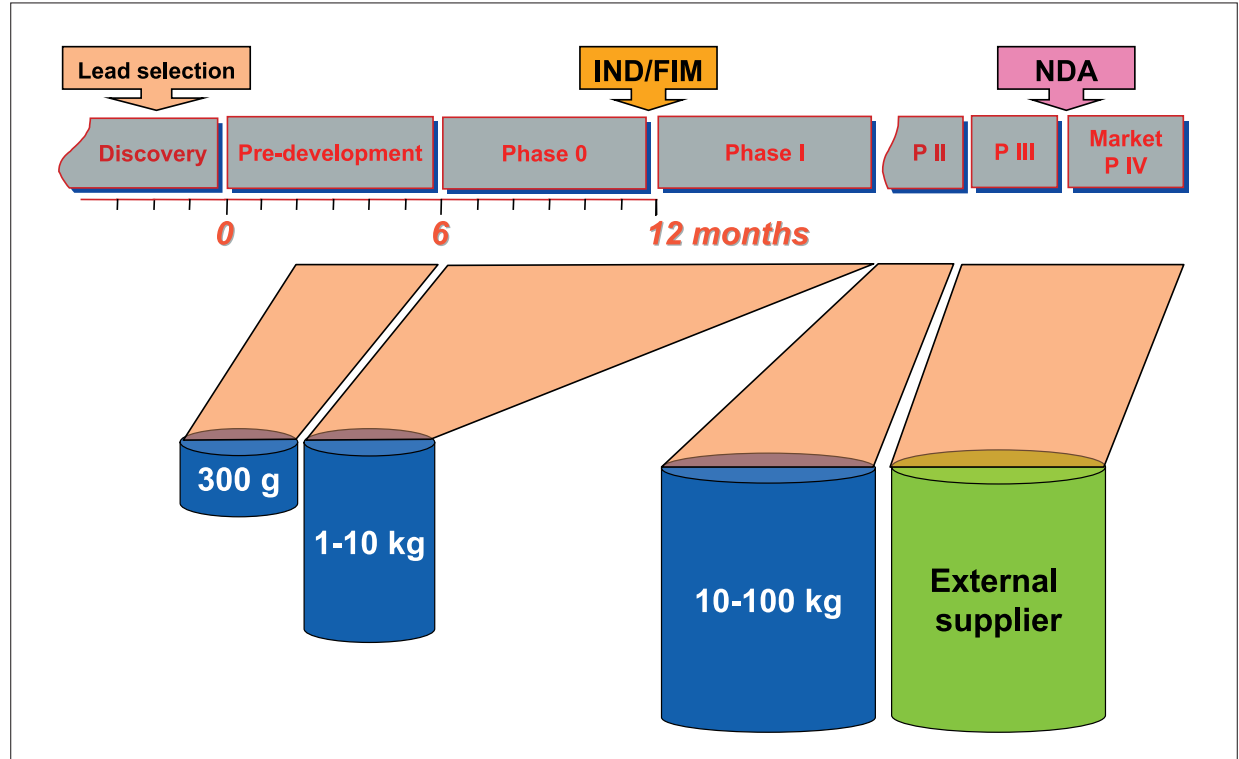

Fig. 1. The timeframes in which the individual scale-up batches are used are indicated by the shaded trapezoids.

addition, changes in the paradigm for preclinical development reduced the end of Discovery phase to IND submission cycletime from 18 months to 12 months. Concurrently, an internal decision to deliver a single drug substance batch supporting both the Phase 0 IND-enabling drug safety and Phase I clinical studies occurred with a consequent need for larger quantities to be delivered earlier.

In response to the Wyeth productivity model, the increased number of compounds in the developmental pipeline, the shortened cycle-time for Phase 0 compounds, and the single-batch paradigm, we needed to modify our approach to early process research and development. A proportionate increase in resources alone did not seem to be a fitting solution. Our new way of working is the subject of this article.

\section{Background and Deliverables}

In order to set the stage, we should familiarize the reader with the unique practices of Wyeth Chemical Development. Fig. 1 depicts our scale-up paradigm, which reveals four discrete stages. Usually, the first three (in blue) are executed in-house; the fourth one (in green) is almost exclusively out-sourced to an external supplier. It is this 
latter aspect of our model that makes Wyeth distinct; it has served our company well and has proven to be cost-efficient and highly operational.

To sustain Wyeth's productivity model (two NDAs every year), about 25 Pre-development compounds must enter Chemical Development annually and 15 projects need to advance to Phase 0 . Within two months of the Pre-development declaration, a smallscale drug substance batch of about $300 \mathrm{~g}$ is prepared for early-stage drug safety studies and formulation development. Shortly thereafter, kilogram-quantities must be delivered to support Phase 0 studies as well as Phase I clinical trials. This kilogram-scale batch is produced in the appropriate cGMP environment and delivered just prior to the Phase 0 decision point, i.e., six months after the Pre-development declaration. Phase I clinical trials begin six months later or 12 months after the end of the Discovery Phase. During Phase I, we prepare 10 to 100 $\mathrm{kg}$ of drug substance needed for additional drug safety work and Phase II clinical studies. Once the compound shows clinical efficacy, we generally out-source any further production to an external supplier.

As depicted in Fig. 2, Synthesis Research \& Development (SRD), the Kg-laboratories, and the Pilot Plant are our in-house scale-up and process research and development functions. SRD prepares the small-scale 300-g batch. They also develop scalable chemistry and transfer the process to the $\mathrm{Kg}$-laboratory for the production of Phase 0/Phase I drug substance. Frequently, Pilot Plant resources are called upon for the scale-up of early steps in the process when volume requirements outstrip the capacity of the Kg-laboratories. Production of Phase II drug substance supplies is the responsibility of the Pilot Plant. Our Sourcing group is instrumental in obtaining starting materials and overseeing the contract manufacturing of drug substance supply at external suppliers.

All early process research and developmentactivities are coordinated through SRD, which is composed of Process Chemistry and Technology functions. Process Chemistry is responsible for process research (i.e., route selection), process development and optimization, early scale-up, and the technology transfer to the internal and external suppliers. The Technology function supports the activities of the process chemists and is comprised of two groups: the high-throughput automation group that we have named InPACT (Integrated Parallel Automation \& Chemistry Technologies) and the CET (Chemical Engineering Technologies) group (Fig. 2). The InPACT group uses state-of-the-art technologies for parallel screening and optimization of reaction parameters as well as for other research and development activities that require their specialized technical expertise.

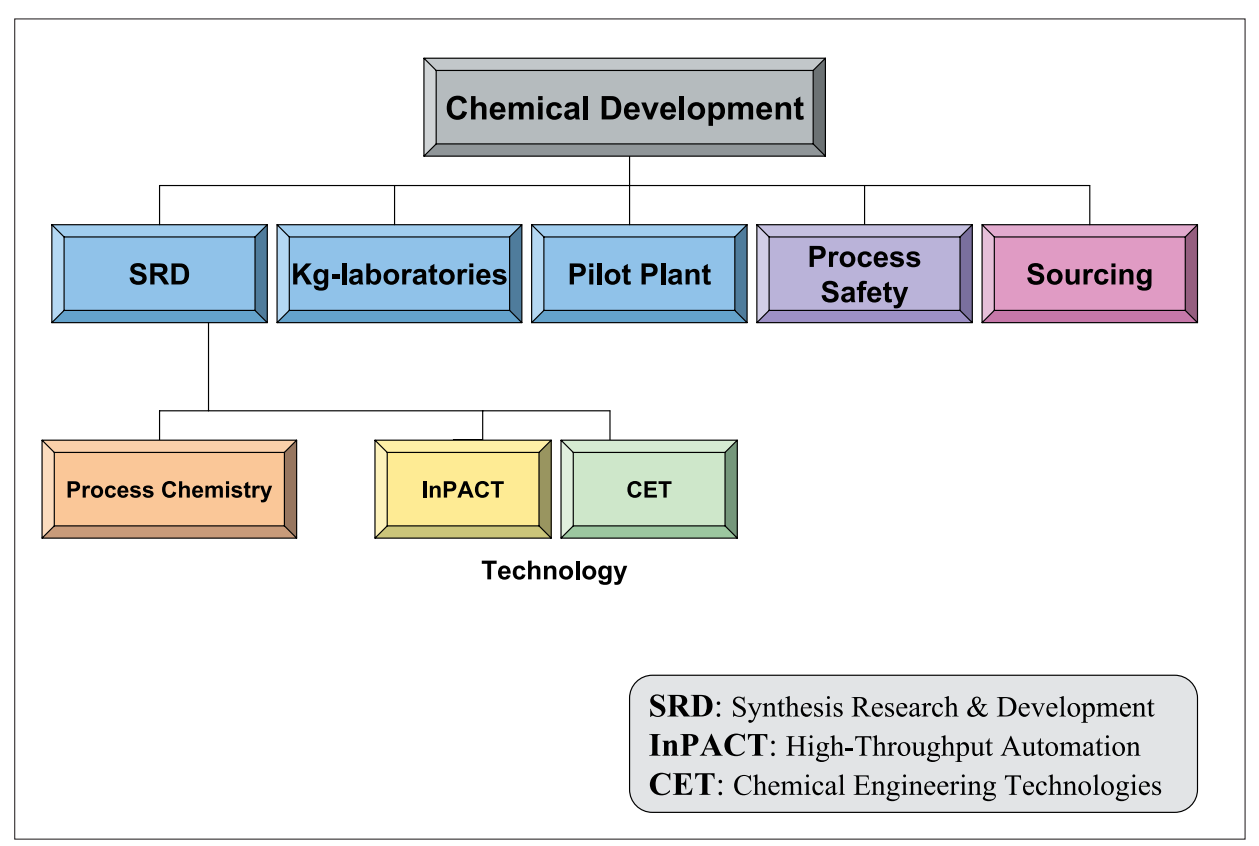

Fig. 2.

The CET group works to understand and improve the physical attributes of the drug substance. They also collaborate with the process chemists to develop unit operations that impact drug substance quality and process scalability. CET is involved over the lifetime of the project, beginning with the selection of a stable form (salt, polymorph, particle morphology and size) and continuing through optimization of crystallization and isolation processes for commercial supply from external suppliers.

In order to ensure the appropriate safety environment, our Safety group provides input regarding process safety and EHS (Environmental Health \& Safety) decision-making for all process R\&D activities whether executed by SRD, the Kg-laboratories or the Pilot Plant. Once compound scale-up has been out-sourced, Sourcing acts as project management and coordinates the logistics and the contractual and business-related issues associated with process development and scale-up of the compound at the external supplier.

The scope of this paper will be limited to our early activities, i.e., route selection, process development, scale-up, and compound deliveries that lead up to the submission of an IND application. In as much as these activities set the direction for later scale-up, additional process $R \& D$ will of course be needed for the eventual commercial preparation of drug substance, however, our post Phase I development activities will not be described at this time.

\section{The Scale-up Deliverables}

Prior to lead selection, Discovery prepares $5 \mathrm{~g}$ of several target compounds for single dose pharmacokinetic studies. Once a single lead has been identified, drug substance requirements increase and Discovery initiates a 25 -g campaign for ascending dose pharmacokinetic studies. A technology transfer from Discovery to Chemical Development occurs at this point. This is followed by a laboratory assessment of the Discovery route together with an in-depth planning exercise, a laboratory proof of concept of suggested route/process changes, scale-up to produce $300 \mathrm{~g}$ of compound for toxicology studies, a technical transfer to the scale-up unit, and finally a $1-10-\mathrm{kg}$ campaign for the combined Phase 0/I supplies (Fig. 3).

\section{The Paradigm}

Beginning with the technology transfer from Discovery and culminating in the delivery of the $1-10-\mathrm{kg}$ batch, our activities proceed through three distinct phases: Plan \& Learn, Learn Scale-up, and Scale-up Confirm (Fig. 3). Gates were established to ensure that activities at the various time points in the development cycle are harmonized across the department and checklists and templates are provided to the teams to enable consistent documentation at each step. The gates are the Initial Discovery Meeting (IDM, Gate 1), the Synthesis Strategy Planning meeting (SSP, Gate 2), the Rapid Synthesis Evaluation meeting (RSE, Gate 3), the Lessons Learned meeting for the small-scale batch (LL, Gate 4), and the Lessons Learned meeting for the $1-10-\mathrm{kg}$ cGMP batch (LL, Gate 5) (Fig. 3).

The success of our early process development model will be driven by a combination of judicious decision-making by sci- 


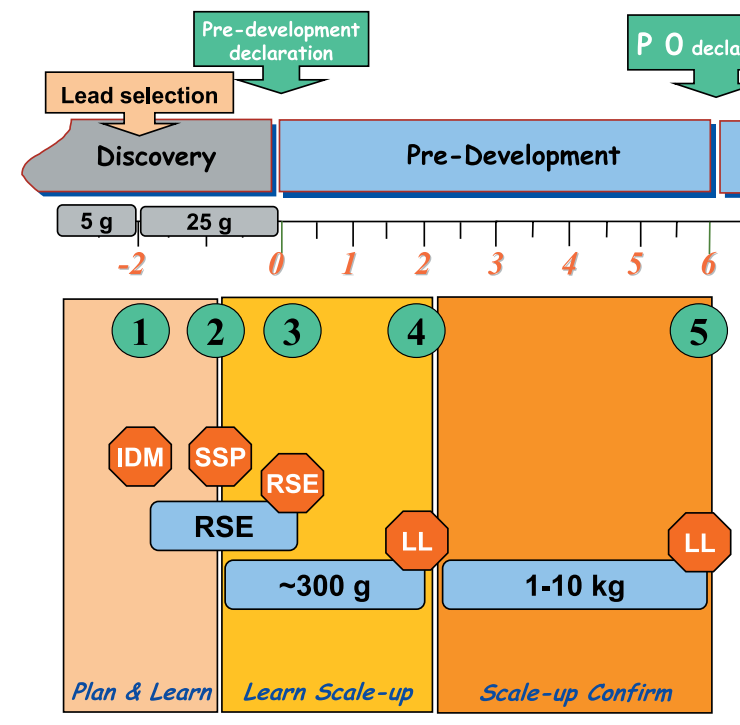

Fig. 3.

entists who understand the complexity of scale and appreciate the downstream production paradigm, together with a system such as the one described below that allows the most efficient use of finite resources.

\section{The Plan \& Learn Phase}

During the Plan \& Learn phase and with the start of the Discovery 5-g synthesis, we get our first look at structures and synthetic routes of compounds we might be asked to scale-up. However, because prior to the IDM the lead candidate has not yet been chosen, there is no Chemical Development core team in place to facilitate the exchange of information between Discovery and us. To ensure good communication between the two groups and to convey rapidly Medicinal Chemistry decisions regarding the advancement (or termination) of leads, we assigned a single scientist to oversee the associated activities, a role referred to as the Interface function. A biweekly meeting was established as the main venue for information exchange with representation from Discovery and Sourcing. Sourcing assists Discovery in raw material procurement. This also benefits Chemical Development by giving advance notice when starting materials are not commercially available; a signal that there is a need for additional laboratory work on our part. Information collected during the meeting is forwarded to the SRD technical leaders who will solicit feedback from the entire organization. Towards this end, eRoom $^{\circledR}$ technology, a web-accessible collaboration tool that enables teams to work together virtually or on-line, is used extensively to facilitate the electronic dissemination, review, and archiving of information. Brainstorming is initiated through either face-to-face or virtual discussions. Concurrently, the CET group will begin selection activities for potential lead candidates, particularly in instances when there are concerns regarding the crystalline properties of these compounds. The resources of the InPACT group are made available to Discovery to support their research and development efforts. Of course this is a win/win proposition as it gives us some experience with Discovery routes of synthesis for potential lead candidates with minimal expenditure of our resources due to the judicious application of automated high throughput technologies.

Lead selection triggers the formation of a Chemical Development core team, comprised of scientists from Process Chemistry, InPACT, CET and Sourcing. Information flow and activities previously directed by the Interface function now become the responsibility of the core team. This team is responsible for scheduling the IDM, a meeting that brings together Discovery, SRD, Sourcing, and the Analytical group to review the process data from the 5-g preparation. Generally, the meeting is short and focused on a Discovery presentation of their synthetic approaches followed by a discussion of practical details such as work-up, process excursions and the like. The physical properties of the compound are reviewed in detail, a strategy to achieve the optimal drug substance form is agreed upon and CET initiates laboratory activities utilizing material supplied by our Discovery colleagues.

The conclusion of the IDM, i.e., passing Gate 1, marks the beginning of a criti- cal stage in the Wyeth R\&D paradigm, the Rapid Synthesis Evaluation (RSE). Conceptually, this is a dual paper and laboratory research exercise that begins about halfway through the Plan \& Learn phase. The laboratory exercise is an in-depth assessment of the Discovery 5-g process. The outcome of the exercise is a better understanding of the details of the chemistry and the identification of scale-up concerns such as hazardous operations, low throughput processes, reactions with solubility issues and high cost or unavailable starting materials. At each step, permutations of the Discovery reaction conditions are studied with 'manual' multi-well screening tools, and process modifications are investigated/implemented in conjunction with this screening process. From the outset, process chemists partner with the InPACT group to identify reactions and operations that would benefit from technologically more sophisticated automated high throughput screening tools or non-perturbational reaction probing. Ultimately, the laboratory evaluation dissects each transformation for operability at scale and provides a basis for determining what is practicable and when alternative approaches should be considered. In cases where it has been determined $a$ priori that the Discovery route or aspects thereof are impracticable at even moderate scale, this evaluation will be omitted in favor of a direct laboratory assessment of alternative chemistries.

The non-laboratory analysis occurs in parallel to the laboratory assessment. Alternative synthetic approaches based on literature precedent/in-house prior knowledge and opportunities to exploit technology options such as continuous flow, simulated moving bed chromatography and the like are researched at this time. Again, eRoom ${ }^{\circledR}$ technology is utilized to assist in the sharing of information throughout Chemical Development and specifically SRD. This vehicle allows ideas, questions, literature citations and comments to be posted in a web-based system, facilitating a more interactive communication amongst our scientists. Typically, this phase of the RSE is completed within three to four weeks of the technical transfer from Discovery. SRD scientists then assemble the data/results for dissemination and review by the Chemical Development core team that has now been expanded to include members of the $\mathrm{Kg}$ laboratories, Process Safety, and the Analytical group. This, the second gate of our process, is referred to as the SSP meeting. During the meeting the RSE results are reviewed, process concerns are discussed and scale-up needs, such as quantities of starting materials and projected batch sizes are assessed. In order to assist in the prioritization of the development work, major issues that could be 'showstoppers' for the upcom- 
ing campaign are identified as 'red flags'. Whenever possible we avoid process development activities that only address short term needs. However, there are occasions when time constraints force us to focus resources on quick fixes that most likely will need to be revisited in the short time leading up to the kg-scale campaign. Questions regarding the strategic direction and degree of thoroughness necessary for the upcoming development work are considered at this time. In the end, a strategy is agreed upon that assures the delivery of a process to carry us through the $1-10-\mathrm{kg}$ campaign while still enabling us to address as many of the long-term issues as possible. The SSP meeting marks the end of the Plan \& Learn phase and the transition to the Learn Scaleup phase.

\section{The Learn Scale-up Phase}

Now that we have gained a reasonable appreciation for the lead's structural and synthetic complexity, we are ready to implement the strategy formulated in the SSP meeting. In the early part of the Learn Scale-up phase the RSE transitions from a Discovery route assessment to an evaluation of the intended route of synthesis and/ or promising alternative approaches. The primary goal is to develop and demonstrate implementable options in a short period of time. The optimization work is carried out in parallel to the synthesis research work, and, in order to conserve precious starting materials, is typically run at very small scale. Intermediates that are isolated during the course of the laboratory experimentation serve as a source of substrates for the InPACT group. The ability of the InPACT group to screen reagents and reaction conditions rapidly and in parallel to the process chemists' optimization routine is essential to our ability to make informed decisions quickly on the appropriate route and process. The RSE exercise targets to provide about $10 \mathrm{~g}$ of penultimate and/or final compound for utilization by the CET group to continue the selection process for the most appropriate stable physical form (salt, polymorph and particle size). In addition, the process chemists will isolate significant quantities of late-stage intermediates to support our objective of focusing process development resources on the value-added last steps. Historically, the routes entering Wyeth's development pipeline average nine steps and approximately $40 \%$ of the compounds contain at least one stereogenic center. Besides the obvious benefit regarding the prioritization of resources, the 'last-steps-early' philosophy is advantageous for a number of other reasons. Since the drug substance impurity profile is affected most by the later stages of a process, it is paramount that we thorough- ly comprehend and have control of these operations. Recently published quality by design (QbD) initiatives underscore the importance of understanding the operating space for the steps that will most likely impact the drug substance impurity profile and physical quality. Towards this end, we first prioritize the criticality of each of the later steps with regard to their impact on the purity of the compound through a quality risk assessment. Then, we investigate the link between drug substance quality and input variables (starting materials and reagents) and process parameters with an eye toward the determination and implementation of an analytical control strategy. Opportunities for Process Analytical Technology (PAT) are considered at this point.

As noted earlier, a very close collaboration is established between the process chemists and the InPACT group at the outset of the RSE. This collaboration is especially valuable as applied to molecules containing stereogenic centers. Because of the increasing number of compounds coming into development that are in this category it was imperative for us to focus internal resources to develop expertise in the field of asymmetric catalysis. Thus, we established a small group within SRD that we refer to as the Chiral Hydrogenation Team (CHT). This team includes scientists from both the Process Chemistry and InPACT groups and begins its involvement soon after the IDM. Their mission is to identify compounds that would benefit from the application of catalytic chiral hydrogenation technologies, identify and synthesize prochiral substrates, assemble an extensive library of chiral ligands, screen commercially available catalysts and ligands, and assist in the incorporation of promising results into processes capable of delivering stereochemically pure bulk drug substance at large scale.

By the time the RSE has been completed, a six to eight week exercise all together, a transition from activities heavily focused on learning to activities that support the scale-up campaign for the 300-g delivery occurs. At the RSE meeting, the third gate in this model, all information collected during the previous weeks is compiled and shared with the expanded Chemical Development core team. Progress is reviewed and modifications to the plan conceived at the SSP meeting are made. Since the RSE meeting occurs one to two months prior to the start of the 1-10 Kg-laboratory campaign, decisions regarding the intended synthesis route must be made at this juncture to allow the starting materials to be ordered. As a consequence of our timelines, we must order the starting materials at risk and prior to the completion of the 300-g campaign, based solely on the experience of the RSE. The need for Kg-laboratory support is also discussed, as it is often advantageous to use the larger vessels for the early steps and/or for steps with throughput issues. Of course this has a profound impact on the number of batches that must be run. Particular attention is given to a review of the results from the value-added last steps of the process to ensure that we have sufficient understanding of the link between input/processing parameters and drug substance quality. Guidelines and a checklist are available in the eRoom ${ }^{\circledR}$ to ensure harmonization in the way the chemists and engineers work at this stage across all projects. Our analytical control strategy and previously considered PAT options are also reviewed. Finally, if relevant, alternative approaches developed by the CHT group are presented and their potential for implementation prior to the $\mathrm{kg}$-scale campaign is assessed.

The RSE meeting is the de facto transition point from learning to the scale-up segment of the Learn Scale-up phase, although in reality, the early steps of the 300-g campaign will most likely already be underway. The process chemists scale-up their reactions in 2-30 L cylindrical jacketed glass reactors that have been designed such that any collected data is reasonably predictive for scale-up facilities. Because reaction volumes are now much greater than previously handled, the active involvement of the Process Safety group relative to hazards analysis is critical to safe laboratory operability. The Kg-laboratories together with Process Safety review each step of the process to minimize surprises at the intended scale. Thermal hazards screening is integral to the process. Prior to the delivery of the 300-g batch, the CET group will have, in conjunction with Pharmaceutical Sciences, completed their drug substance physical form selection process (salts and polymorphs). The InPACT group continues to work in parallel with the process chemists, bringing cutting edge technology like the ReactIR $^{\circledR}$ and the power of automated 24/7 robotics like the Anachem ReactArray ${ }^{\circledR}$ to bear on problematic steps. Reactor systems such as the Mettler Toledo LabMax ${ }^{\circledR}$ have proven to be invaluable as 'scale-down' tools. Continuous, close communication between SRD and the Kg-laboratories and Pilot Plant over the course of the 300-g campaign is essential, and open dialogue regarding unsuccessful reactions and unexpected results saves us headaches later on. Issues related to mixing, rate and order of addition of reagents, thermal excursions, exaggeration of impurities on preliminary scale-up, and serendipitous crystallizations are shared to help to pave the way for the transfer of the process.

Following successful delivery and release of the 300-g batch, a Lessons Learned meeting is convened, which marks our fourth gate and the transition to the Scaleup Confirm phase. In this meeting, the ex- 
panded Chemical Development core team reviews the process and delineates a plan for its transfer to and ultimate scale-up in the Kg-laboratories. Parallel work activities are considered, i.e., alternative component route research, an updated work plan is created and the process control strategy is reviewed and updated as necessary. Items such as the need for elimination of solvents previously chosen for expediency (e.g., methylene chloride) or the feasibility of implementation of an asymmetric hydrogenation approach in place of a low yielding resolution are also discussed. Output from the meeting is archived in the eRoom ${ }^{\circledR}$ as we transition into the final phase of our early process development model.

\section{The Scale-up Confirm Phase}

In the past, delivery of the small-scale batch preceded the start of the $1-10-\mathrm{kg}$ campaign by at least two to four months and process chemists used this time to address scale-up problems. In our current paradigm this luxury no longer exists since the largerscale campaign must begin at the time the 300 -g batch is delivered, if not earlier. To ease the abrupt transition, early involvement on the parts of the Kg-laboratories and Process Safety is necessary and therefore they are part of the Chemical Development core team from the time of the SSP meeting, i.e., Gate 2. Chemists and chemical engineers will have identified operational and safety issues early so that the process chemists can address them in parallel to the preparation of the small-scale batch. This buys time, allowing a more rapid transition of the small-scale process to the Kg-laboratories and helps to ensure a greater success rate. Corrosion concerns and the need for specialized equipment, such as flow reactors, are also considered early and dealt with proactively. Approximate reaction and work-up throughputs are the basis of preliminary calculations that identify potential bottlenecks regarding equipment available in our Kg-laboratories as well as to decide if Pilot Plant help is needed for early steps. Batch reactions are modified to semi-batch as needed to allow for safe operation and control of highly exothermic reactions. All raw materials and intermediates are tested for thermal stability and their safety is assessed at the intended operating conditions. Further hazards analysis and testing is done as the process becomes better defined and a formal 'what-if' hazards and operability (HAZOP) review is conducted before start of the actual batch.

A Standard Laboratory Procedure, written by the SRD scientists, is the core of the technical package transferred to the Kg-laboratory. A draft procedure for a given step is reviewed for safety and operability and is the basis for a laboratory demonstration of the process carried out by the process chemists in the presence of Kg-laboratory personnel. The process is run at a minimum 1-L scale in jacketed cylindrical reactors. Video streaming units have been installed that allow remote, yet interactive viewing of the process by scientists and engineers without being obliged to travel to the site where the process is demonstrated. Generating data on phase splits, filtrations, distillations, mixing, and crystallization profiles is critical as the $\mathrm{Kg}$-laboratory personnel use the Standard Laboratory Procedure together with their own observations to generate a Master Batch Record for the largescale campaign. The draft Master Batch Record and corresponding process flow diagram are reviewed with SRD chemists and Process Safety at a HAZOP/batch record review meeting. After a satisfactory review, the batch operation can begin with an SRD chemist observing and serving the role of troubleshooter/advisor.

Another Lessons Learned meeting, the final gate of this phase of development, is held after the completion of the 1-10 kg cGMP campaign. The purpose of the meeting is to review information gathered for the individual steps (yield and quality) executed in the Kg-laboratory and to compare this to the prior laboratory demonstration runs. The data and results are reviewed in order to prioritize additional process development work that is considered necessary for the success of future campaigns. Decisions regarding the question of process optimization versus alternative strategies may not be made at this time but a plan of attack will be discussed. In as much as the Kg-laboratory Lessons Learned meeting marks the end of the early stage of development at Wyeth, it is the stepping off point for later stage de- velopment efforts geared towards supporting the successful implementation of the process at incrementally larger scales and ultimately, if the project moves forward, launch.

\section{Conclusion}

\section{"How times have changed!"}

Prompted by the implementation of the Wyeth productivity model five years ago, we, in Chemical Development, ended up revising our way of doing business entirely. Timelines and the traditional sequence of process development and scale-up activities, which had proven successful in the past, were discarded and new concepts put in place. However, revised timelines and processes alone don't necessarily lead to improved productivity and efficiency. Only with the continuous support of our chemists and engineers, together with their openness to implement change, could we ensure the success of the above-described paradigm for early-stage route selection, process development, scale-up, and drug substance deliveries.

As the reader might suspect, the abovedescribed model did not come about all at once. It was the result of multiple changes, which were designed, evaluated, and implemented over time that finally gelled to achieve our current process. The fruits of all these changes have still not been fully harvested, and many of our projects have not yet taken advantage of all aspects of this prototype for early process development in Wyeth. However, the expansion in the number of projects Chemical Development now advances from Discovery into Phase I speaks for itself. We really do bring about four times as many more projects into IND stage than we did five years ago, without a commensurate increase in resources. This simple fact, we believe, would have been unattainable without the implementation of the early development process described above. In the final analysis, the success has to be attributed to the attitude of our motivated chemists and engineers who brought this paradigm to life.

Received: June 17, 2006 